\title{
Boundary Conditions for Jet Flow Computations
}

\author{
M. E. Hayder \\ Institute for Computational Mechanics in Propulsion \\ NASA Lewis Research Center \\ Cleveland, $\mathrm{OH} 44135$ \\ and \\ Eli Turkel \\ Institute for Computational Mechanics in Propulsion \\ NASA Lewis Research Center \\ Cleveland, $\mathrm{OH} 44135$ \\ and \\ Department of Mathematics \\ Tel-A viv University \\ Tel-Aviv, Israel
}

\begin{abstract}
Ongoing activities (Mankbadi et al ${ }^{1}$ ) are focused on capturing the sound source in a supersonic jet through careful LES. One issue that is addressed herein is the effect of the boundary conditions, both inflow and outflow, on the predicted flow fluctuations, which represent the sound source. In this study, we examine the accuracy of several boundary conditions to determine their suitability for computations of time-dependent flows. Various boundary conditions are used to compute the flow field of a laminar axisymmetric jet excited at the inflow by a disturbance given by the corresponding eigenfunction of the linearized stability equations. We solve the full time dependent Navier-Stokes equations by a high order numerical scheme. For very small excitations, the computed growth of the modes closely corresponds to that predicted by the linear theory. We then vary the excitation level to see the effect of the boundary conditions in the nonlinear flow regime.
\end{abstract}

Copyright (C)1994 by the American Institute of Aeronautics and Astronautics, Inc. No copyright is asserted in the United States under Title 17, U.S. Code. The U.S. Government has a royalty-free license to exercise all rights under the copyright claimed herein for Governmental purposes. All other rights are reserved by the copyright owner.

\section{Basic Scheme}

We use a high order extension of MacCormack's scheme, due to Gottlieb and Turkel ${ }^{2}$, to solve the Navier-Stokes equations. For the two dimensional Navier-Stokes computations, the operator $L$ in the equation $\mathrm{L} Q=\mathrm{S}$ or equivalently $Q_{t}+F_{x}+G_{\tau}=S$ is split into two one-dimensional operators and the scheme is applied to these split operators. For the one dimension model/split equation $Q_{t}+F_{z}=S$, we express the predictor step with forward differences as

$\bar{Q}_{i}=Q_{i}^{n}+\frac{\Delta t}{6 \Delta z}\left\{7\left(F_{i+1}^{n}-F_{i}^{n}\right)-\left(F_{i+2}^{n}-F_{i+1}^{n}\right)\right\}+\Delta t S_{i}$

and the corrector step with backward differences as

$$
Q_{i}^{n+1}=\frac{1}{2}\left[\bar{Q}_{i}+Q_{i}^{n}\right.
$$

$$
\left.+\frac{\Delta t}{6 \Delta z}\left\{7\left(\bar{F}_{i}-\bar{F}_{i-1}\right)-\left(\bar{F}_{i-1}-\bar{F}_{i-2}\right)\right\}+\Delta t S_{i}\right]
$$

This scheme is second order in time and becomes fourth-order accurate in the spatial derivatives when alternated with symmetric variants 23 . We define $L_{1}$ as a one dimensional operator with a forward difference in the predictor and a backward difference in corrector. Its symmetric variant $L_{2}$ uses a backward difference in predictor and a forward difference in the corrector. 
For our computations, the one dimensional sweeps are arranged as

$$
\begin{gathered}
Q^{n+1}=L_{1 z} L_{1 r} Q^{n} \\
Q^{n+2}=L_{2 r} L_{2 z} Q^{n+1}
\end{gathered}
$$

This scheme is used for the interior points. In order to advance the scheme near boundaries the fluxes are extrapolated outside the domain to artificial points using a cubic extrapolation to compute the solution on the boundary. We then solve equation (2.1) to get the solution at the new time for all boundary points. Hence, in all one dimensional sweeps, the equations are updated based on the extrapolated fluxes when necessary. At the completion of a time step, i.e. a predictor and corrector in both one dimensional sweeps, the boundary condition is imposed at both inflow, outflow and the top boundary. For some boundary conditions, at outflow, we found it better to include the boundary equation in all the $z$ sweeps. This will be indicated in the next section. The singularity at $r=0$, is eliminated by using L'hospital's rule. We have used an axisymmetric code and also a three dimensional code in axisymmetric mode for the computations presented in this paper. Extrapolations at the bottom boundary are used for the computations with the three dimensional code. The top boundary is a characteristic boundary and so is treated differently than the outflow boundary. We extrapolate three characteristic variables from the interior and set $p_{t}-\rho c v_{t}=0$. For most of the cases we used zeroth order extrapolation, but we also considered the effect of linear extrapolation. In this study we concentrate on the effect of the outflow boundary condition and, to a lesser extent, on the inflow boundary treatment.

\section{Outflow Boundary Conditions}

We base the boundary treatment on the inviscid equation, even though we solve the viscous equations. This is reasonable since the viscous effects at inflow and outflow are negligible for high Reynolds number flows. There have been many studies 345678 attempting to minimize the reflections at the outflow boundary. Our present study is a continuation of previous works 38 . The main difficulty occurs for subsonic flows. For supersonic flows, all the characteristics travel in the flow direction and boundary points can be calculated from known quantities at inflow or by extrapolation from the interior at outflow. For subsonic flow, one acoustic wave propagates against the flow direction. One condition is needed corresponding to this characteristic variable. The simplest approach is to linearize the equations and to use the one dimensional characteristic variables normal to the surface. One then specifies the incoming characteristic variables and extrapolates the outgoing variables. Characteristic variables can also be obtained by solving differential equations instead of by extrapolation. For the acoustic waves one needs differential equations for $p_{t} \pm \rho c u_{t}$, where $u$ is the velocity component normal to the boundary. For the shear wave one needs $v_{t}$, where $v$ is tangential to the boundary and finally $p_{t}-c^{2} \rho_{t}$ for the entropy variable. In this study, whenever the boundary condition is not specified but is free to float then the appropriate characteristic variable is updated by the partial differential equation (except when mentioned explicitly). In order to avoid one sided differences the fluxes are extrapolated outside the domain to artificial points using a cubic extrapolation.

Whenever the appropriate combination is specified we replace this by specifying the combination of the time derivatives. We can describe this as

$$
\begin{gathered}
p_{t}-\rho c u_{t}=R_{1} \\
p_{t}+\rho c u_{t}=R_{2} \\
p_{t}-c^{2} \rho_{t}=R_{3} \\
v_{t}=R_{4}
\end{gathered}
$$

where $R_{i}$ is determined by which variables are specified and which are not. Whenever, the combination is not specified, $R_{i}$ is just those spatial derivatives that come from the Navier-Stokes equations. Thus, $R_{i}$ contains viscous contributions even though the basic format is based on inviscid characteristic theory. In implementing these differential equations we convert them to conservation variables $\rho, \mathrm{m}=\rho \mathbf{u}, \mathrm{n}=\rho \mathrm{v}$ and $\mathrm{E}$. Assuming an ideal gas we then have

$$
\begin{gathered}
p_{t}=(\gamma-1)\left(E_{t}+\frac{u^{2}+v^{2}}{2} \rho_{t}-u m_{t}-v n_{t}\right) \\
u_{t}=\frac{m_{t}}{\rho}-\frac{u \rho_{t}}{\rho} \\
v_{t}=\frac{n_{t}}{\rho}-\frac{v \rho_{t}}{\rho}
\end{gathered}
$$

For subsonic outflow we calculate $R_{2}, R_{3}, R_{4}$ from the Navier-Stokes equations and set $R_{1}$ as prescribed by the given boundary condition. For supersonic flows, all the $R_{i}$ at the outflow boundary can be calculated from the Navier-Stokes equations or else by extrapolation of all the characteristic variables from the interior.

\subsubsection{Scott-Hankey condition}

Scott and Hankey ${ }^{9}$ developed a condition to specify the incoming characteristic variable at the outflow, for the computation of unsteady flow in a transonic 
compressor rotor. Characteristic variables $K_{1}$ to $K_{4}$ are defined as

$$
\begin{gathered}
K_{1}=\frac{p}{\rho_{\infty} c_{\infty}}-u \\
K_{2}=\frac{p}{\rho_{\infty} c_{\infty}}+u \\
K_{3}=\rho-\frac{p}{c_{\infty}^{2}} \\
K_{4}=v
\end{gathered}
$$

At the outflow boundary $K_{2}, K_{3}$ and $K_{4}$ are extrapolated from interior using

$$
\begin{aligned}
& \frac{\partial K_{2}}{\partial z}=0 \\
& \frac{\partial K_{3}}{\partial z}=0 \\
& \frac{\partial K_{4}}{\partial z}=0
\end{aligned}
$$

$K_{1}$ is specified at the exit boundary using $p_{e x i t}, u_{e x i t}$, $p_{\infty}$ and $u_{\infty}$. pexit and $u_{e x i t}$ are imposed in the inviscid region and their axial derivatives are assumed to be zero in the viscous region. For this implementation, as in Scott et al. ${ }^{8}$, we assume $p_{\text {exit }}$ and $u_{\text {exit }}$ to be $99 \%$ of their inflow values at the small radial locations. For large radial locations $p_{e x i t}$ and $u_{e x i t}$ are assumed to be their values at the immediate interior point in the axial direction. We do not solve equation (2.1) when the Scott-Hankey condition is imposed.

\subsubsection{One dimensional characteristic condition}

The one dimensional nonreflecting (characteristic) condition is $R_{1}=0$. This condition implies that the time derivative of the amplitude of the incoming characteristic wave is zero. This case is equivalent to the nonreflecting condition proposed by Thompson ${ }^{5}$. We have used this condition in previous studies 1310 . For this case we found it more accurate to evaluate the boundary condition within the $\mathrm{z}$ sweeps, both predictor and corrector, rather than at the end of the entire time step. We refer to this implementation as the Thompson condition.

\subsubsection{Giles' Condition}

Giles $^{6}$ (and later Kroner ${ }^{11}$ ) added some tangential space derivatives in the outflow condition. He considers a wave-like solution of the form

$$
U(x, y, t)=e^{i(k x+l y-\omega t)} u^{r}
$$

The boundary condition is derived by constructing a row vector $\nu_{n}^{L}$ such that $\nu_{n}^{L} U=0$ for each $\mathrm{n}$ corresponding to the incoming wave. Here we consider his second order, two-dimensional, unsteady outfiow condition. The equation for $R_{1}$ then becomes

$$
p_{t}-\rho c u_{t}=-u \rho c v_{y}-v\left(p_{y}-\rho c u_{y}\right)=R_{1}
$$

In polar coordinate we implement this as

$$
p_{t}-\rho c u_{t}=-u \rho c \frac{(r v)_{r}}{r}-v\left(\frac{(r p)_{r}}{r}-\rho c \frac{(r u)_{r}}{r}\right)=R_{1}
$$

This has the advantage of using only tangential derivatives at the outflow boundary which are discretized by central differences.

\subsubsection{Bayliss-Turkel condition}

Based on an asymptotic form of the wave equation Bayliss and Turkel ${ }^{7}$ derived a nonreflecting condition. They then used a change of variables to consider the case of a nonzero mean flow. Let $d^{2}=\frac{x^{2}}{1-\|^{2}}+y^{2}$ where $M$ is the Mach number. $x, y$ are the physical locations of the boundary point relative to some source. For jet flows this source is taken as the inflow. For other flows an appropriate center must be chosen. There is no reason, in general, to measure $z$ and $r$ from the center of the local coordinate system. Then they got

$$
\begin{aligned}
& \frac{1}{c \sqrt{1-M^{2}}}\left[1-\left(\frac{x}{d}\right) \frac{M}{\sqrt{1-M^{2}}}\right] p_{t} \\
& +\left(\frac{x}{d}\right) p_{x}+\left(\frac{y}{d}\right) p_{y}+\frac{p-p_{\infty}}{2 d}=0
\end{aligned}
$$

In three dimensions the inhomogeneous term is divided by $d$ rather than the $2 d$ (see two and three dimensional conditions by Roe ${ }^{12}$ ). For cylindrical coordinates $(r, \theta, z)$ multiply by $\frac{d}{T}$ and use $x=r \cos (\theta), y=r \sin (\theta)$ and the definition of $\mathrm{d}$ to get

$$
\frac{s(\theta)}{c} p_{t}+\cos (\theta) p_{x}+\sin (\theta) p_{y}+\frac{p-p_{\infty}}{2 \tau}=0
$$

where

$$
s(\theta)=\frac{\sqrt{1-M^{2} \sin ^{2}(\theta)}-M \cos (\theta)}{1-M^{2}}
$$

Hariharan and Hagstrom ${ }^{13}$ derived (2.1.4) as a boundary condition and noted its equivalence to the BaylissTurkel condition (see also $§ 2.1 .7$ ). Bayliss and Turkel then used the momentum equations to get $p_{x}$ and $p_{y}$ in terms of $u_{t}$ and $v_{t}$ and other spatial derivatives of $u$ and $v$. Also assuming that $v_{\infty}=0$ this yields

$$
\begin{gathered}
p_{t}-p c \frac{x}{d \sqrt{1-M^{2}}}\left(u_{t}-u v_{y}\right)+c \sqrt{1-M^{2}} \frac{y}{d} p_{y} \\
+\frac{c \sqrt{1-M^{2}}}{2 d}\left(p-p_{\infty}\right)=0
\end{gathered}
$$


For many cases, the domain is much longer than it is high. Using $y<<x$ we have a simplified form

$$
p_{t}-\rho c u_{t}=-\rho c u v_{y}=R_{1}
$$

In polar coordinates, we write the above equation as

$$
p_{t}-\rho c u_{t}=-\rho c u \frac{(r v)_{\tau}}{r}=R_{1}
$$

With this simplification we get an equation for the characteristic variable in a form similar to that proposed by Giles ${ }^{6}$ but not identical with his condition. We will refer this condition as the simplified Bayliss-Turkel condition.

We also consider another less simplified form of Bayliss-Turkel boundary condition. We implemented this condition in our present axisymmetric jet computations as

$$
\begin{gathered}
p_{t}-\rho c u_{t}=-\rho c u \frac{(r v)_{r}}{r}-c \sqrt{1-M^{2}} \frac{(r p)_{r}}{d} \\
+\frac{c \sqrt{1-M^{2}}}{d}\left(p-p_{\infty}\right)=R_{1}
\end{gathered}
$$

In this study, we refer to this condition as the Bayliss-Turkel condition. The spatial derivatives are approximated by central differences.

\subsubsection{Riemann variables condition}

Hagstrom and Hariharan ${ }^{14}$ derived a boundary condition by coupling the incoming and outgoing Riemann variables, $a_{1}$ and $a_{2}$

$$
\begin{aligned}
& a_{1}=u+\frac{2 c}{\gamma-1} \\
& a_{2}=u-\frac{2 c}{\gamma-1}
\end{aligned}
$$

where $a_{1}$ corresponds to the outgoing acoustic wave and $a_{2}$ corresponds to the incoming acoustic wave. Using asymptotic analysis in the far field in cylindrical coordinates one gets

$$
\left(u-\frac{2 c}{\gamma-1}\right)_{t}=\frac{c_{\infty}}{2 z}\left[u-\frac{2\left(c-c_{\infty}\right)}{\gamma-1}\right]=R_{1}
$$

For this case we found it more accurate to evaluate the boundary condition within the z sweeps, both predictor and corrector, rather than at the end of the entire time step.

Instead of their full condition, we implement a one dimensional version and use the above equation to calculate $R_{1}$. For convenience, we replace the equation for $R_{2}$ with

$$
\left(u+\frac{2 c}{\gamma-1}\right)_{t}=R_{2}
$$

It should be noted that this boundary condition was derived with the assumption that $u_{\infty}=0$. As $z \rightarrow \infty$, $R_{1} \rightarrow 0$ and this condition reduces to an one dimensional characteristic condition equivalent to the one in \$2.1.2. Since $c$ at the outflow has a profile we choose the constant $c_{\infty}$ based on the outflow at the top of the domain. The poor results for this scheme may be due to these choices for $u_{\infty}$ and $c_{\infty}$ and because we implemented a truncated version.

\subsubsection{Roe's condition}

Roe ${ }^{12}$ derived one boundary condition from the bi-characteristic equations as

$$
\begin{gathered}
\left(x-\beta M_{\infty} d\right) u_{t}+\beta^{3} d c_{\infty} u_{x}+\beta^{2} y\left[v_{t}+u_{\infty} v_{x}\right] \\
+c_{\infty}\left(\beta d-M_{\infty} x\right) v_{y}=\frac{p-p_{\infty}}{2 \rho_{\infty}}
\end{gathered}
$$

where $\beta=\sqrt{1-M_{\infty}^{2}}$ and $\mathrm{d}$ as defined in $\$ 2.1 .4$. This condition replaces the equation for $R_{1}$. For $x>y$, the above equation reduces to

$$
u_{t}+c_{\infty}\left[\left(1+M_{\infty}\right) u_{x}+v_{y}\right]=\frac{p-p_{\infty}}{2 x \rho_{\infty}\left(1-M_{\infty}\right)}
$$

We further simply this equation by dropping the inhomogeneous term to get

$$
u_{t}+c_{\infty}\left[\left(1+M_{\infty}\right) u_{x}+v_{y}\right]=0
$$

We implemented this condition and found the solution near the outflow to be oscillatory. We, then made the following modification to improve this condition. We use the energy equation with $v=0$

$$
p_{t}+u p_{x}+\rho c^{2}\left(u_{x}+v_{y}\right)=0
$$

to convert equation $(2.1 .6)$ to

$$
p_{t}-\rho c u_{t}=-u p_{x}+\rho c^{2} M u_{x}=R_{1}
$$

The $x$ derivatives are calculated by one sided differences. We shall refer to this as Roe's condition.

\subsubsection{Convective pressure condition}

As pointed out before, Bayliss and Turkel developed a radiation condition for the wave equation based on an asymptotic form. This was generalized to the convective wave equation by a change of variables. Bayliss and Turkel boundary condition was derived for two dimension. Their pressure boundary condition was. extended to three dimension by Roe ${ }^{12}$. Later Hariharan and Hagstrom ${ }^{13}$ developed an asymptotic expansion directly for the convective wave equation based on 
a Riemann function. Thus, in cylindrical coordinates $(r, \theta, z)$ with $R^{2}=r^{2}+z^{2}$

$$
p \sim \sum_{j=0}^{\infty} \frac{F_{j}\left(t-\frac{R}{U(\theta)}, \theta\right)}{R^{j+1}}
$$

where

$$
U(\theta)=u_{\infty} \cos \theta+c_{\infty}\left(1-M^{2} \sin \theta\right)^{1 / 2}
$$

and $M=\frac{u_{\infty}}{c_{\infty}}$. $U$ is the inverse of $s(\theta)$ as given in $\$ 2.1 .4$. This gave them a set of boundary conditions identical to the complete Bayliss-Turkel conditions and in addition they were now able to prove uniform convergence to get improved error estimates. Tam and Webb ${ }^{15}$ considered the linearized Euler equations. Using Fourier and Laplace transforms they independently derived the first term of the asymptotic expansion for the convective wave equation. They then derived an outflow boundary condition based on the far field asymptotic solutions of the linearized Euler equations. Hence, the approaches of Bayliss-Turkel, Hariharan-Hagstrom and Tam-Webb all yield the same equation. This is given in two dimensions by (2.1.4). While we have recast the nonreflecting boundary conditions in characteristic form, Tam and Webb considered eq. (2.1.4) as an equation for the pressure and supplemented it by the linearized Euler equations for the density and the velocities. We have tried using the characteristic formulation with three dimensional extension of equation (2.1.4) [i.e., factor $2 \mathrm{r}$ is replaced by $r$, but the formulation suggested by Tam and Webb gave reduced oscillations at the outfiow.

$$
\text { In 3-D spherical coordinates, }
$$

$(R, \theta, \phi)$, this boundary condition is given by

$$
\frac{p_{t}}{U}+p_{R}+\left(p-p_{\infty}\right) / R=0
$$

We next transform from spherical to cylindrical coordinates. Then $p_{R}=p_{r} \cos (\theta)+p_{z} \sin (\theta)$ where $\sin (\theta)=$ $\frac{z}{R}$ (note this is different than the cylindrical coordinate $\theta$ previously used). In this study, we consider a long domain with small height i.e. $r<<z$ or equivalently $R \sim z, \sin (\theta) \sim 1$. Thus,

$$
p_{t}+(u+c) p_{z}+\frac{p-p_{\infty}}{z}=0
$$

where $u$ is the axial velocity at the outfiow boundary. In this paper we will refer this condition as the simplified convective pressure condition.

\subsubsection{Filtering}

Besides nonreflecting boundary conditions based on the partial differential equation there are other approaches to remove the difficulties associated with far field boundaries. One such approach was used by Colonius et al. ${ }^{16}$, who introduced a filter near the outflow. In this study, we examine the effect of filters for unsteady flow calculations. We choose a sixth order filter of the form

$$
\begin{aligned}
& \bar{f}_{i}=(1-a) f_{i}+a\left[\frac{11}{16} f_{i}+\frac{15}{64}\left(f_{i+1}+f_{i-1}\right)\right. \\
& \left.-\frac{3}{32}\left(f_{i+2}+f_{i-2}\right)+\frac{1}{64}\left(f_{i+3}+f_{i-3}\right)\right]
\end{aligned}
$$

where $a=0$ at the beginning of the filter(exit) zone and increases linearly to 1 at the outflow boundary.

\section{Inflow Boundary Condition}

For supersonic flow all characteristic variables propagate in the flow direction and one can specify all the flow variables at the inflow. For subsonic flow, one characteristic variable propagates against the flow direction and it needs to be extrapolated or estimated from the interior. Other conditions may be specified by the given inflow conditions. We specified the appropriate given inflow boundary conditions as the mean field plus a constant $\epsilon$ times a time varying part based on the linear spatial stability theory eigenfunctions. If $\epsilon$ is sufficiently small we expect the full Navier-Stokes equations to behave similar to the linear theory, at least for small distances from the inflow boundary.

\subsection{BCI1: Extrapolate $p-p_{0} c_{0} u$}

In this treatment, we extrapolate one characteristic variable (corresponding to the out going acoustic wave) from the interior and specify three conditions based on inflow conditions. We then use these four conditions to update all quantities at the inflow. In particular, we extrapolate $\left(p-\rho_{0} c_{0} u\right)$ from interior. We impose $v$ and calculate $\left(p+\rho_{0} c_{0} u\right)$ and $\left(p-c_{0}^{2} \rho\right)$ from the given inflow condition. $p_{0}$ and $c_{0}$ are the linearized density and speed of sound at the inflow.

\subsection{BCI2:}

In this case the inflow boundary condition is imposed using the equation (2.1). We calculate $R_{1}$ from the solution of the Navier-Stokes equation at the inflow and calculate $R_{2-4}$ from the given inflow condition. We then solve the above set of equations to get corrected time derivatives.

\subsection{BCI3:}

Instead of specifying $\mathrm{v}$ as in the previous section we can specify the normal derivative $v_{x}$. Since for small $\epsilon$

$$
v=\epsilon e^{\alpha z} \operatorname{Re}\left[V(r) e^{i \omega t}\right]
$$


$v_{z}$ at inflow $(z=0)$ is given by

$$
v_{z}=\epsilon \alpha \operatorname{Re}\left[V(r) e^{i \omega t}\right]
$$

This boundary condition is weaker in that we supply derivative information rather than the $v$ component directly and so $v$ can shift by a function of time. On the other hand we are supplying additional information on the growth rate $\alpha$ which is lacking in the other boundary conditions. When the linear eigenfunction is not known one can sometimes specify the vorticity as suggested by Roe ${ }^{12}$.

\section{Linear Stability Theory}

Linear stability theory is used to compute eigen functions and the growth rate of disturbance modes. For completeness of this paper, we will briefly discuss this theory. The disturbances, which are governed by the linearized Euler equations, are assumed to be spatially growing and time harmonic with a monochromatic frequency. This form of the disturbance in axisymmetric polar coordinates is given by

$$
[u, v, p, \rho]=[\hat{u}, \hat{v}, \hat{p}, \hat{\rho}] e^{i(\alpha z-\omega t)}+\text { complex conjugate }
$$

The radian frequency $\omega$ is a real quantity while the eigenvalue $(\alpha)$ is a complex quantity. The disturbance equations can be reduced to a single equation for the pressure amplitude ${ }^{17}$ given by

$$
P_{r r}+\left[\frac{1}{r}-\frac{W_{r}}{W}\right] P_{\tau}-\left[\alpha^{2}\left(1-M^{2} W\right)+\frac{N^{2}}{r}\right] P=0
$$

where

$$
W=\left[U-\frac{\omega}{\alpha}\right]^{2} \rho_{0}
$$

The rest of the disturbance functions are given by

$$
\begin{gathered}
\rho \hat{v}=\frac{i}{\alpha(U-C)} P_{r} \\
\rho \hat{u}=-\frac{1}{\alpha^{2}(U-c)^{2}} P_{r} U_{r}-\frac{p}{U-C} \\
\hat{\rho}=-\frac{1}{\rho \alpha^{2}(U-C)^{2}} \rho_{r} P_{r}+\frac{P}{c^{2}}
\end{gathered}
$$

where $c$ is the local speed of sound of the jet for with

$$
c^{2}=c_{0}^{2} T
$$

As I goes to zero(jet axis) or to infinity (ambient fluid), $\mathrm{W}$ becomes constant. In this case an asymptotic solution can be obtained in the form

$$
\begin{aligned}
& P \sim I_{N}\left(\alpha \sqrt{1-M^{2} W(0)} r\right) \text { as } r \rightarrow 0 . \\
& P \sim K_{N}\left(\alpha \sqrt{1-M^{2} W(\infty)} r\right) \text { as } r \rightarrow 0 .
\end{aligned}
$$

where $I_{N}$ and $K_{N}$ are modified Bessel function of order $N$. For a given mean flow and a specified frequency, equation (4.1) is solved by iteration for the complex eigenvalue $\alpha$ that satisfies the above boundary condition.

\section{Results}

We first investigate the effect of the outflow boundary conditions for unsteady jet simulations in axisymmetric coordinates $(z, r)$. We excite the flow at the inflow with the eigenfunctions of the linearized stability equations. Because of its importance in sound source computation, we examine the growth of the pressure disturbance downstream. We are interested in a supersonic jet flowing in a subsonic free stream. We vary the infiow excitation and see the effect of the boundary conditions for both the linear and nonlinear regime of the flow.

We consider a jet with the mean inflow profile

$$
\begin{gathered}
\bar{U}_{r}=U_{\infty}+\left(U_{c}-U_{\infty}\right) g_{r} \\
\bar{T}_{r}=T_{c}+\left(T_{\infty}-T_{c}\right) g_{r}+\frac{\gamma-1}{2} M_{c}^{2}\left(1-g_{r}\right) g_{r} \\
g_{r}=\frac{1}{2}\left[1+\tanh \left(\frac{\frac{1}{r}-r}{4 \theta}\right)\right]
\end{gathered}
$$

where $\theta$ is the momentum thickness. The subscripts $c$ and $\infty$ refer to the centerline and free stream values respectively. At inflow, we assume the radial relocity is zero and the static pressure is constant. The standard size of our computational domain is 50 radii in the axial and 5 radii in the radial directions respectively. We excite the inflow profile at location $\mathrm{I}$ and time $\mathrm{t}$ as

$$
\begin{gathered}
U(r, t)=\bar{U}(r)+\epsilon \operatorname{Re}\left(\hat{U} e^{i \pi S_{t} t}\right) \\
P(r, t)=\bar{P}(r)+\epsilon \operatorname{Re}\left(\hat{P} e^{i \pi S_{t} t}\right) \\
\rho(r, t)=\bar{\rho}(r)+\epsilon \operatorname{Re}\left(\hat{\rho} e^{i \pi S_{t} t}\right) \\
V(r, t)=\epsilon \operatorname{Re}\left(\hat{V} e^{i \pi S_{t} t}\right)
\end{gathered}
$$

$\hat{U}, \hat{V}, \hat{\rho}$ and $\hat{P}$ are the eigenfunctions of the linearized equations with the same mean flow profile. $\epsilon$ is the excitation level, $S_{t}$ is the Strouhal number. For small $\epsilon$ the growth of the disturbance modes should agree with that predicted by the linearized Euler equations.

We consider a case with $\frac{U_{\infty}}{U_{c}}=\frac{1}{4}, \frac{T_{\alpha}}{T_{c}}=\frac{1}{2}$, momentum thickness, $\theta=\frac{1}{8}$ and Strouhal number, $S_{t}=\frac{1}{8}$. The jet center Mach number is 1.5 while the Reynolds number based on the jet diameter is 1.2 million. Unless otherwise mentioned, the axial variation of the disturbances correspond to $S_{t}=\frac{1}{8}$ at the jet edge $(\mathrm{r}=1)$. This also corresponds to the excitation frequency in the simulations. Pressure disturbances in this study are calculated by a discrete Fourier transform in time and so they are functions of $\mathrm{z}$.

$$
\left|\hat{P}_{k}\right|=\left|2 \sum_{j=0}^{n-1} P_{j} e^{\frac{i 3 \pi j k}{n}}\right|
$$


We examine the effects of the different outflow conditions presented in $\$ 2$. We use three values of $\epsilon$, $2.5 \times 10^{-4}, 2.5 \times 10^{-3}$ and $5.0 \times 10^{-3}$ and call them low, moderate and high excitation levels respectively. These excitation levels were chosen after numerical experiments to show linear, intermediate and nonlinear effects on the growth of the disturbance. Computations with low level of disturbances were done with a $300 \times 120$ grid. Figure 1.1 compares the prediction of the linear theory with the growth of the pressure disturbance $(|\hat{P}|)$ with different outflow boundary treatment for these computations. $|\hat{P}|$ is the absolute value of the Fourier transform of pressure in time at $S_{t}=\frac{1}{8}$ along the jet edge $(r=1)$. Since the disturbance level is low, as expected computed solutions are close to the prediction of the linear theory. In Figure 1.2 the spectra of the pressure disturbance at the outflow boundary is shown. Only discrete points in $x$ are calculated. They are connected by a straight line only to increase visualization. As shown in Figure 1.1b numerical solutions slightly oscillate and deviate from the prediction of the linear theory. Deviation from the linear theory could come from the nonlinear effects. Boundary treatments could lead to unphysical oscillations, which a high order scheme like 2-4 may retain in the numerical solution.

We next consider computations for $\epsilon=2.5 \times 10^{-3}$ with a $225 \times 150$ grid. $|\hat{P}|$ and the pressure spectra at the outflow boundary are shown in Figures 2.1 and 2.2 respectively. For this $\epsilon$, the boundary condition effects are more noticeable. We see a significant amount of excitation of higher harmonics, i.e., nonlinearity in the spectra of pressure disturbances at the outflow. $|\hat{P}|$ still shows linear growth for this level of excitation. The Riemann variable condition shows more oscillations near the outflow than the other conditions. Finally, we excite the inflow with the high excitation level, $\epsilon=5 \times 10^{-3}$. We compute the numerical solutions both in our standard domain (50 radii long and 5 radii high) and in an extended domain (80 radii long and 5 radii high). We use a $250 \times 100$ grid for the standard domain. $|\hat{P}|$ and the pressure spectra for $x=50$ are shown in Figures 3.1 and 3.2 respectively. For this level of excitation, we observe a decay of pressure oscillations due to nonlinear effects. Comparisons of $|\hat{P}|$ near the outflow boundary show oscillations for all the boundary treatments. In Figure 4 we consider the same case but computed in the extended domain with a $400 \times 100$ grid. In Figures 3.1 and 4.2, we see oscillations away from the outflow boundary. Since different outflow boundary conditions yield essentially identical results at those locations, such oscillations are not likely due to the treatments of outflow boundary. In Figure 5.1 we compare the solutions computed in the standard domain with those from the extended domain. Only the portion of the domain near the outflow boundary is displayed. The figures until now have been based on a zeroth order extrapolation at the upper boundary. We also consider linear extrapolation at the upper boundary. This results in a higher growth rate downstream for all the boundary conditions. Otherwise, the differences between the various boundary treatments is the same as before. The convective pressure, Giles and Thompson conditions were the most accurate near the outflow boundary. We plot only these in Figure 5.2 for both zeroth and linear extrapolation with the higher set of curves representing linear extrapolation.

We next examine the effect of an exit layer. Exit layer approach was used by Colonius et al. ${ }^{16}$. In this study we examine the concept, but use different stretching and filter. We compute three cases. In the first case, we used a $300 \times 120$ grid for the standard domain and added 60 grid points in the axial direction. We refer to this layer $(60 \times 120$ grid points) as the exit layer. We used a $x^{1.5}$ stretching in this exit layer. With the exit layer the computational domain is about 75 radii long and 5 radii high. In the second case, we added a filter (eq. (2.1.8)) in the exit layer. In the third case, we computed the solution in the longer domain but with a $450 \times 120$ grid. We refer this solution as the 'no stretching, no filter' case. We used a low excitation level ( $\epsilon$ $\left.=2.5 \times 10^{-4}\right)$ for the exit layer study. The comparison of $|\hat{P}|$ is shown in Figure 6.1. We observe pressure oscillations introduced by stretching. These oscillations propagate into the interior from the exit layer. With filtering these oscillations are reduced. Similar observations can also be made from the pressure spectra at $x=50$ for these three cases as shown in Figure 6.2.

In figure 7.1 we compare the solutions for three inflow conditions. For conditions BCI1 and BCI2 there is an adjustment region of about 4 jet diameters. The solutions downstream are not affected by this. Nevertheless, we do not expect any adjustment region since we are forcing the solution with the linear eigenfunctions. The width of this adjustment region remains the same when we refine the grid. Hence, this is not a purely numerical artifact. If we specify the normal derivative of $\mathrm{v}$ as given in $\mathrm{BCI} 3$ we considerably reduce this adjustment region but at the expense of increased oscillations in the domain. The improvement when specifying $v=$ may be because we are including the growth rate, $\alpha$, in the boundary condition. We obtained similar results by enforcing vorticity instead of $v$ at the inflow. In figure 7.2 we compare the solutions for different grids for a supersonic jet entering a supersonic ambient flow. In this case all variables are specified at inflow. Nevertheless, we observe a large scale oscillation in the solution about 
the linear growth rate. This indicates that for supersonic flow there are nonlinear effects even at the low perturbations levels. For all the subsonic/supersonic cases considered the solutions were given along the jet edge. Hence, some of the adjustment region may be physical due to the supersonic flow in the jet.

\section{Conclusions}

In the tests performed, for a supersonic jet flow entering a subsonic ambient media, the best outflow boundary condition seems to be the convective pressure condition. Solutions near the outflow were less oscillatory when the boundary condition was supplemented by the linearized Euler equations as suggested by Tam and Webb instead of using the characteristic formulation. The simplifications introduced by Bayliss and Turkel when they derived their final condition reduces the accuracy. The boundary condition suggested by Giles was comparable to that of the convective pressure condition. These boundary conditions were implemented after a complete time step with all intermediate steps using third order extrapolation of the fluxes. Using the differential equations based on one dimensional characteristics was equally good if this was incorporated in all the $\mathrm{z}$ sweeps and not just done at the end of the entire time step. All these conditions closely followed the long domain solution except for small oscillations in the final ten percent of the domain. Hence, one should add about ten percent beyond the domain of interest and then any of these conditions can be used. The other conditions showed larger deviations at the outflow but were still useful. Stretching the mesh at the outflow introduced oscillations which were reduced by filtering.

The inflow for the subsonic/supersonic case exhibited an adjustment region until the linesr growth rate was achieved even though the linear eigenfunctions were used for the inflow data. The size of this adjustment region was mesh independent. For the supersonic/supersonic flow, oscillations were observed further down the jet and also appeared to be independent of the mesh. When the $v_{z}$ (or vorticity) was specified, the results for the supersonic/subsonic flow agreed better with linear theory with a smaller adjustment region. This may be because specifying $v_{z}$ gives additional information about the growth rate. However, because only derivative information is given, the resultant solution was less smooth.

For both inflow and outflow there is need to determine whether it is better to impose the boundary conditions within the sweeps or to impose the boundary conditions only at the conclusion of an entire time step. The boundary condition equations may need to be split to implement within the sweeps. In this study, the one dimensional characteristics (Thompson) and the Riemann variable conditions were both better when used within the sweeps. Both these conditions do not involve any spatial derivatives.

\section{References}

${ }^{1}$ Mankbadi, R. T., Hayder, M. E. and Povinelli, L. A. (1993), "The Structure of Supersonic Jet Flow and Its Radiated Sound", AIAA paper 93-0549.

${ }^{2}$ Gottlieb, D. and Turkel, E. (1976), "Dissipative Two-Four Methods for Time Dependent Problems", Math. Comp, Vol 30, pp 703-723.

${ }^{3}$ Hayder, M. E. and Turkel, E. (1993), " High Order Accurate Solutions of Viscous Problems", AIA A paper 93-3074.

${ }^{4}$ Engquist, B. and Majda, A.(1977), "Absorbing Boundary Conditions for the Numerical Simulation of Waves", Mathematics of Computations, Vol 31, No 139, 629-651.

${ }^{5}$ Thompson, K. W. (1990), "Time Dependent Boundary Conditions for Hyperbolic System, II", Journal of Computational Physics, 89, 439-461.

${ }^{B}$ Giles, M.G. (1990), "Nonreflecting Boundary Conditions for Euler Equations Calculations," AIAA Journal,Vol 28, pp. 2050-2058.

${ }^{7}$ Bayliss, A. and Turkel, E., (1982), "Far Field Boundary Condition for Compressible Flows", Journal of computational Physics, Vol 48, pp 182-199.

${ }^{8}$ Scott, J. N, Mankbadi, R. R., Hayder, M. E. and Hariharan S. I. (1993), "Outflow Boundary Conditions for the Computational Analysis of Jet Noise", AIAA paper 93-4366.

${ }^{8}$ Scott, J. N and Hankey, W. L., (1985), "Boundary Condition for Navier-Stokes Solutions of Unsteady Flow in a Compressor Rotor", Three-Dimensional Flow Phenomena in Fluid Machinery, Vol 32, ASME, New York.

${ }^{10}$ Hayder, M.E. Turkel, E. and Mankbadi, R. R.(1993), "Numerical Simulations of a High Mach Number Jet Flow", AIA A paper 93-0653.

${ }^{11}$ Kroner, D. (1991), "Absorbing Boundary Conditions for the Linearized Euler Equations in 2-D", Math. Comput. 57,pp. 153-167.

${ }^{12}$ Roe, P. L. (1989), "Remote Boundary Conditions for Unsteady Multidimensional Aerodynamic Computations", Computer \& Fluids, Vol 17, No. 1, 221-231

${ }^{13}$ Hariharan, S. I. and Hagstrom, T., (1990), "Far Field Expansion for Anisotropic Wave Equations", Computational Acoustics (IMACS), Vol 2, Lee, Cakmak and Vichnevetsky (Editors), Elsvier Science Publisher, pp 283-294. 
${ }^{14}$ Hagstrom, T. and Hariharan, S. I., (1988), "Accurate Boundary Conditions for Exterior Problems in Gas Dynamics", Mathematics of Computation, Vol 51, No 184, pp 581-592

${ }^{15} \mathrm{Tam}$, C. K. W. and Webb, J. C., (1993),

"Dispersion- Relation- Preserving Finite Difference Schemes for Computational Acoustics", Journal of Computational Physics, 107, pp 262-281.

${ }^{16}$ Colonius, T., Lele, S. K. and Moin, P., "Boundary Conditions for Direct Computations of Aerodynamic Sound Generation", AIAA paper 92-02-075.

17 Michalke, A. (1984), "Survey of Jet Instability Theory", Prog. Aerospace Sci., 21, pp 159-199. 


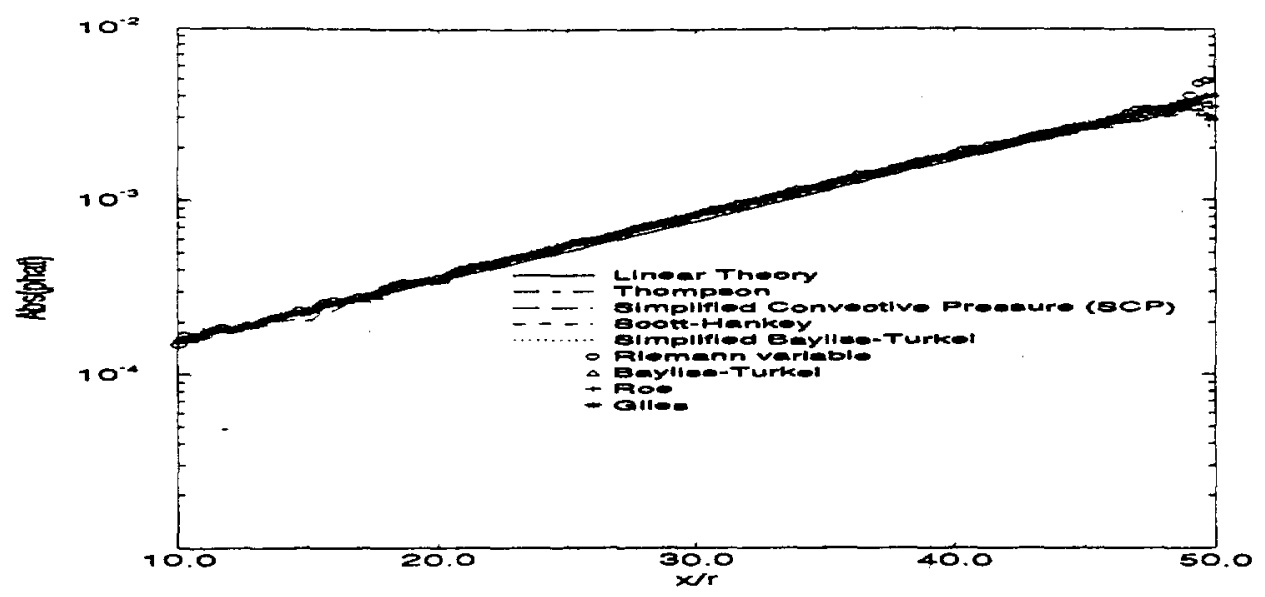

Figure 1.1a: Amplitude of pressure oscillation $(|\hat{P}|)$ for $\epsilon=2.5 \times 10^{-4}$

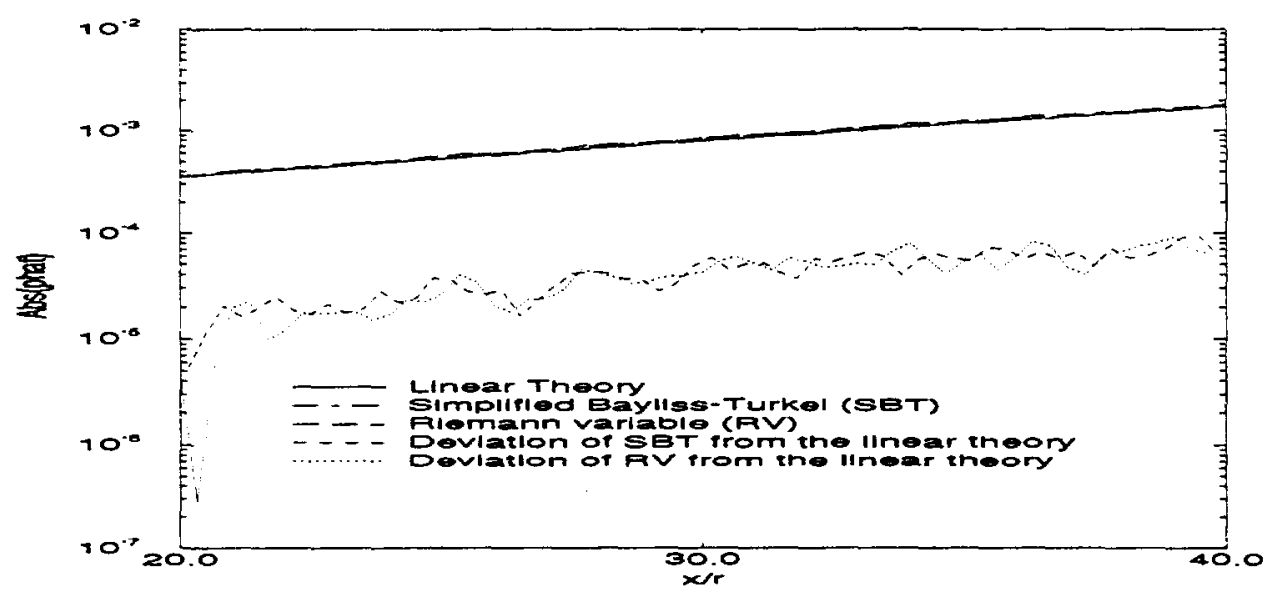

Figure 1.1b: Comparison of the linear theory prediction with numerical solutions

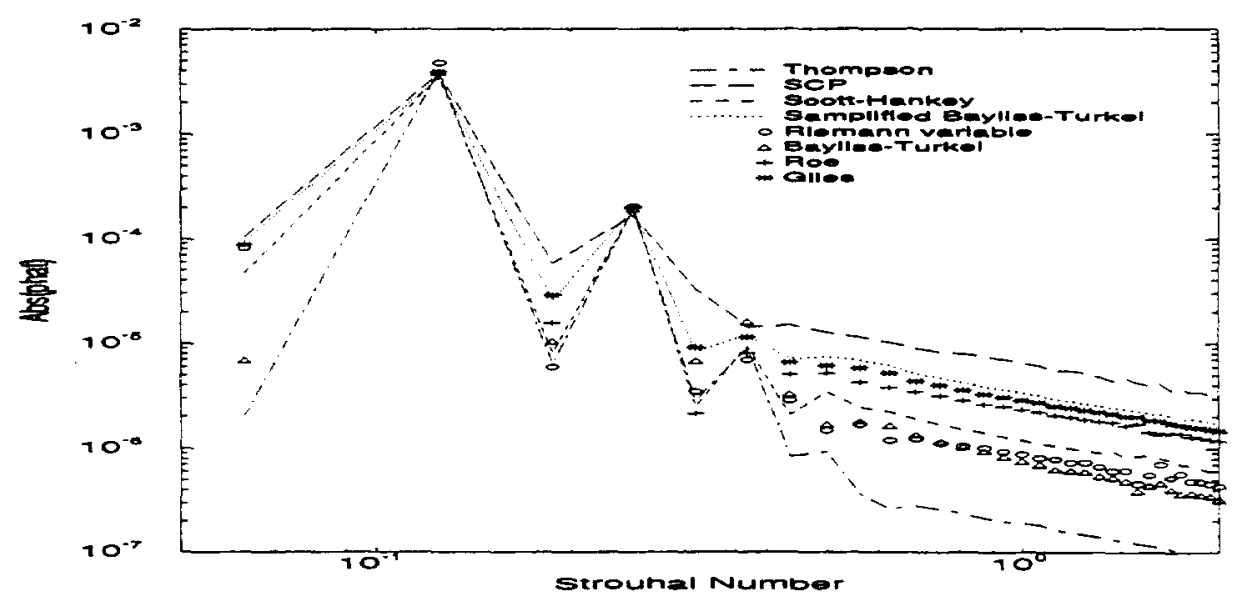

Figure 1.2: Pressure spectra for $\epsilon=2.5 \times 10^{-4}$ 


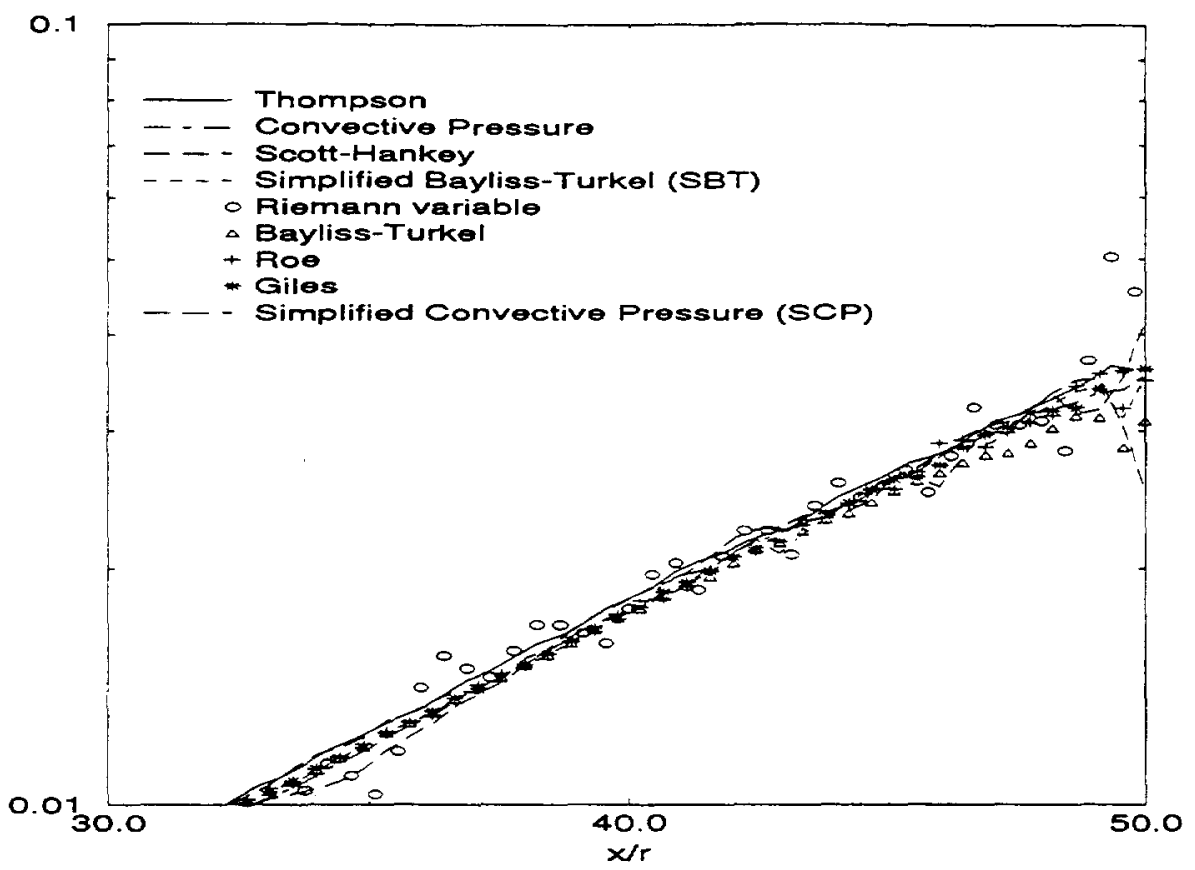

Figure 2.1: Amplitude of pressure oscillation for $\epsilon=2.5 \times 10^{-3}$

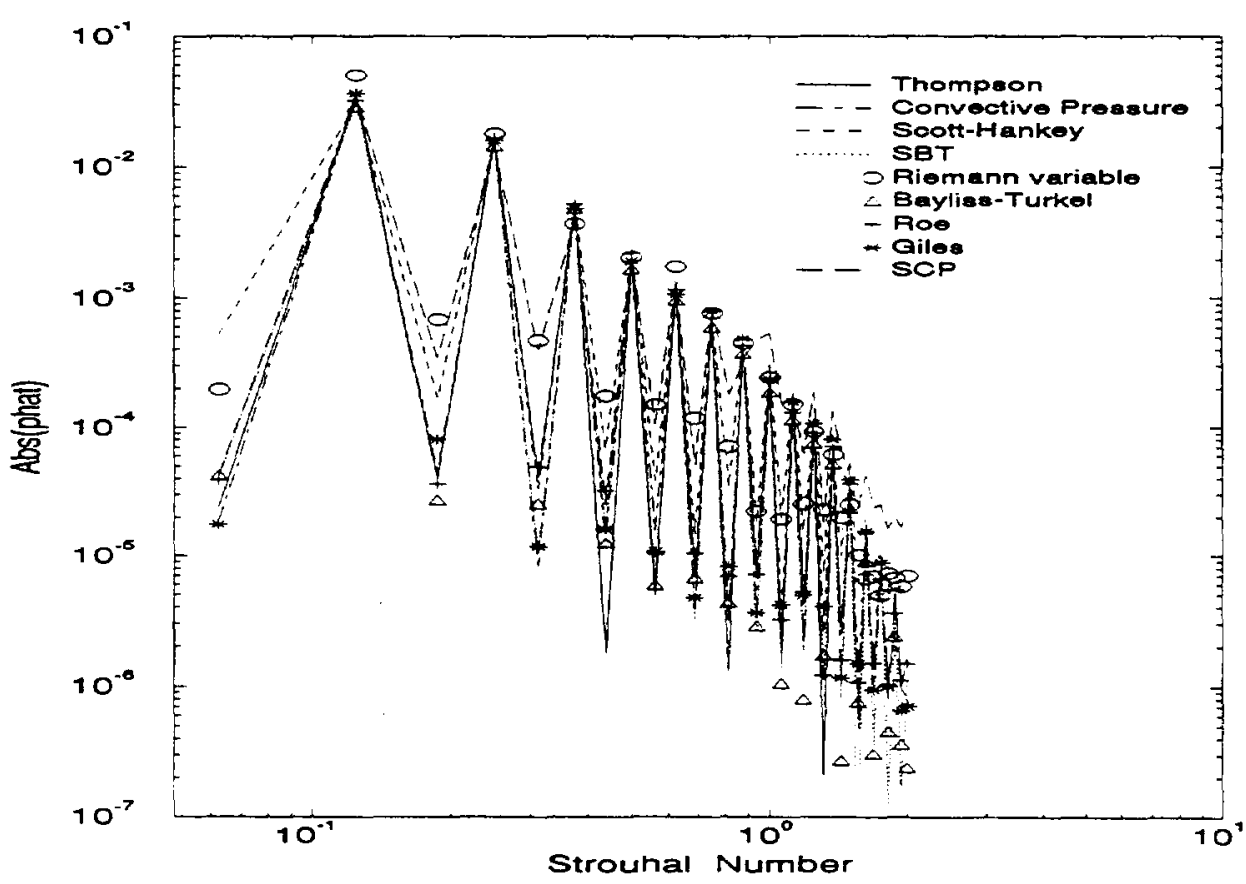

Figure 2.2: Pressure spectra for $\epsilon=2.5 \times 10^{-3}$ 


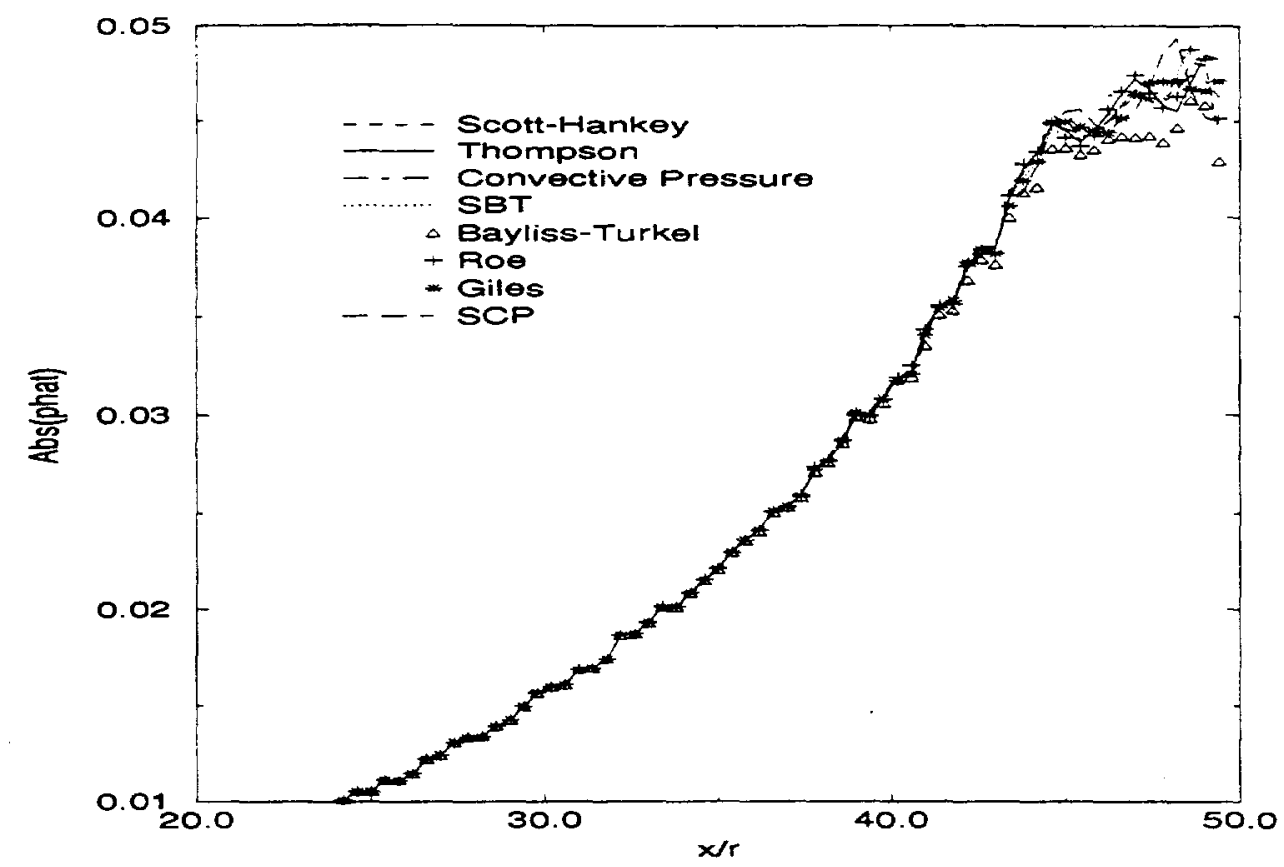

Figure 3.1: Amplitude of pressure oscillation for $\epsilon=5 \times 10^{-3}$

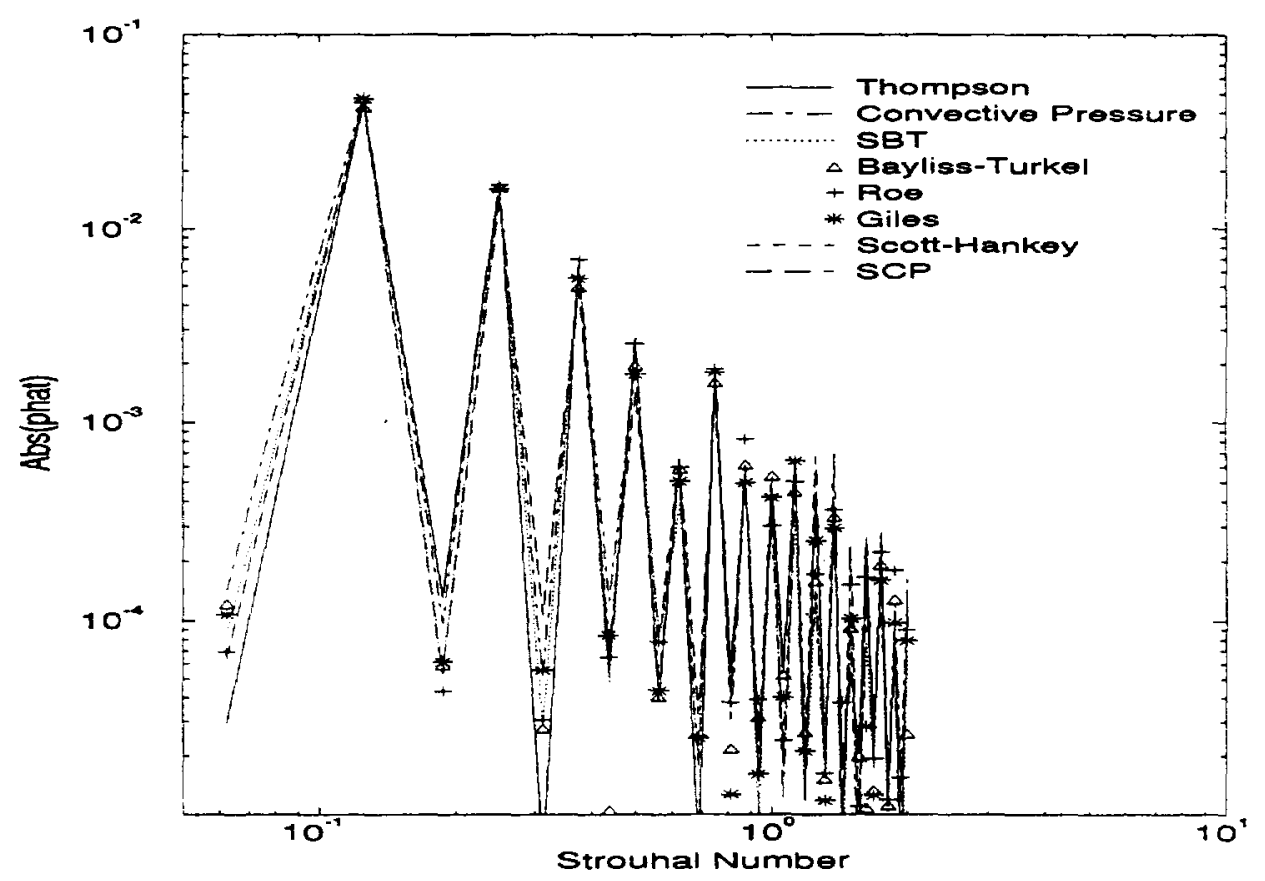

Figure 3.2: Pressure spectra for $\epsilon=2.5 \times 10^{-3}$ 
$100 \times 400$ Grid

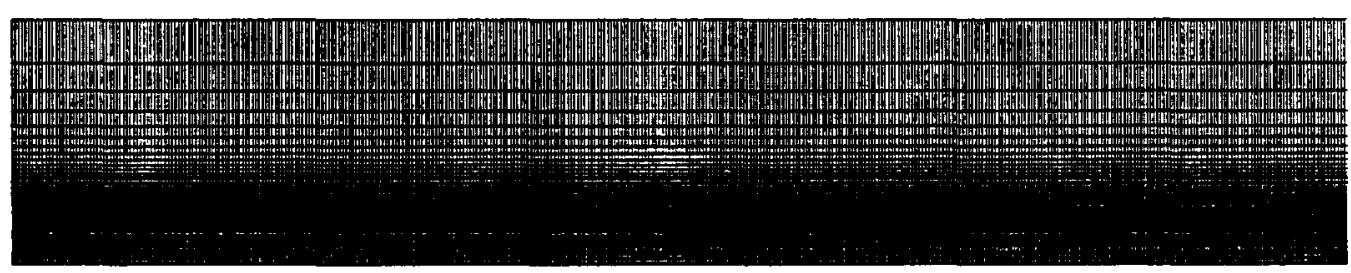

Figure 4.1: Grid for the long domain solution

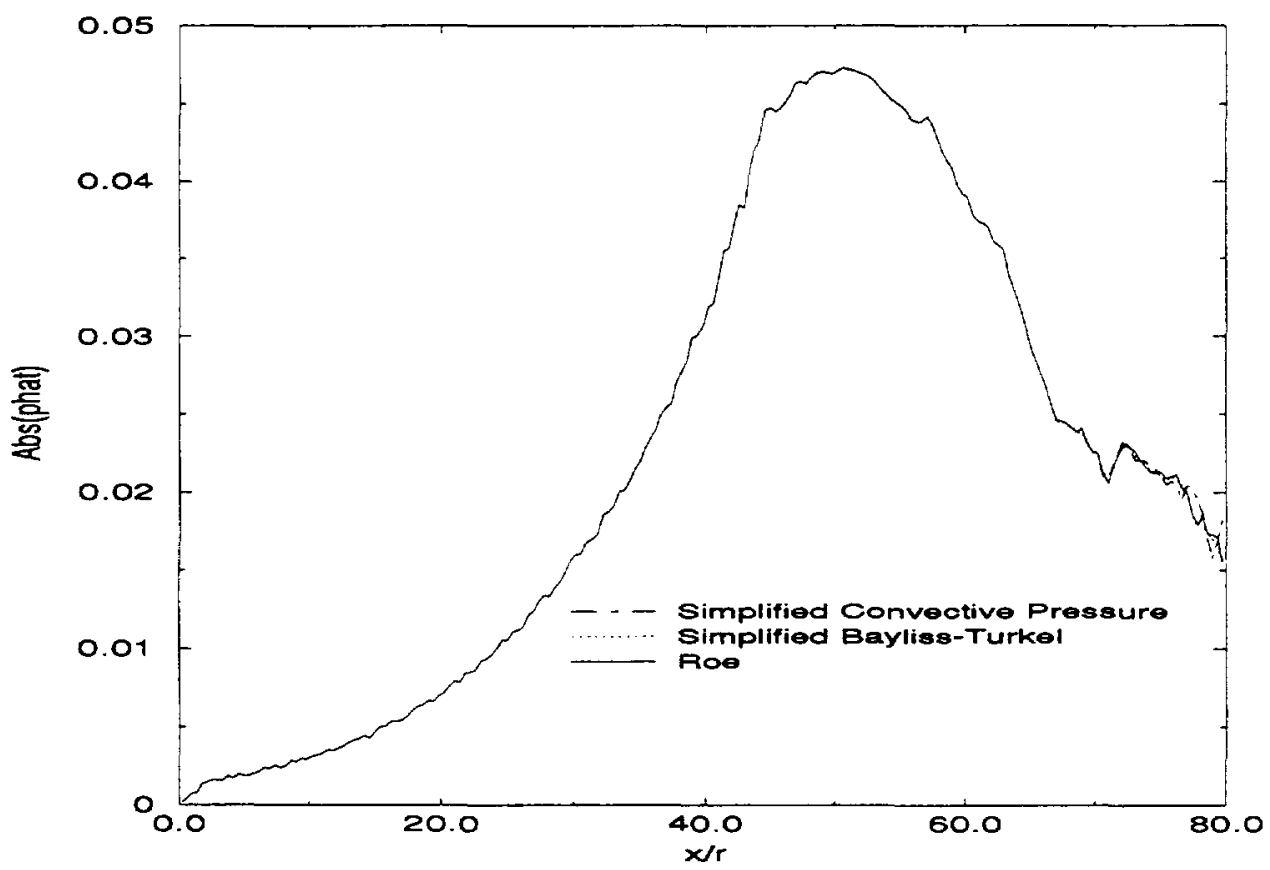

Figure 4.2: Long domain solution for $\epsilon=5 \times 10^{-3}$ 


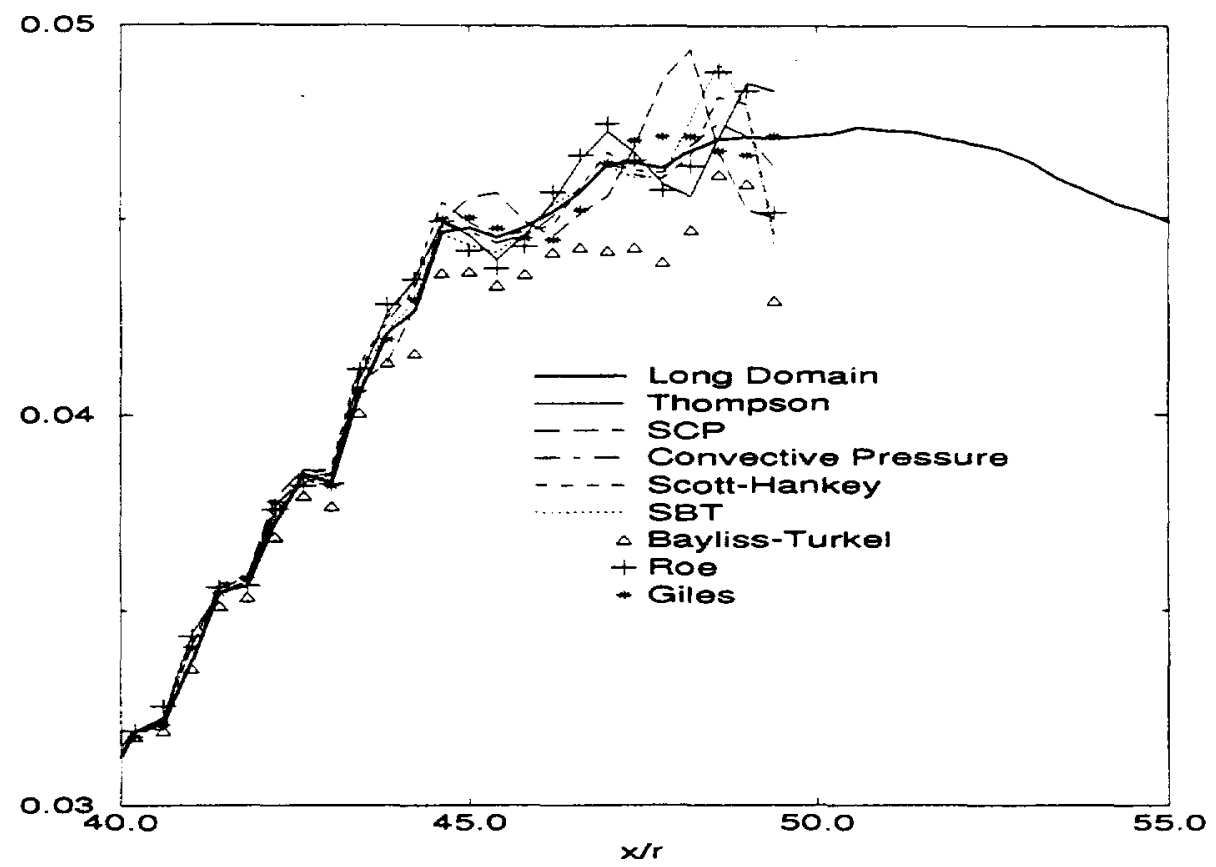

Figure 5.1: Comparison of long and short domain solutions

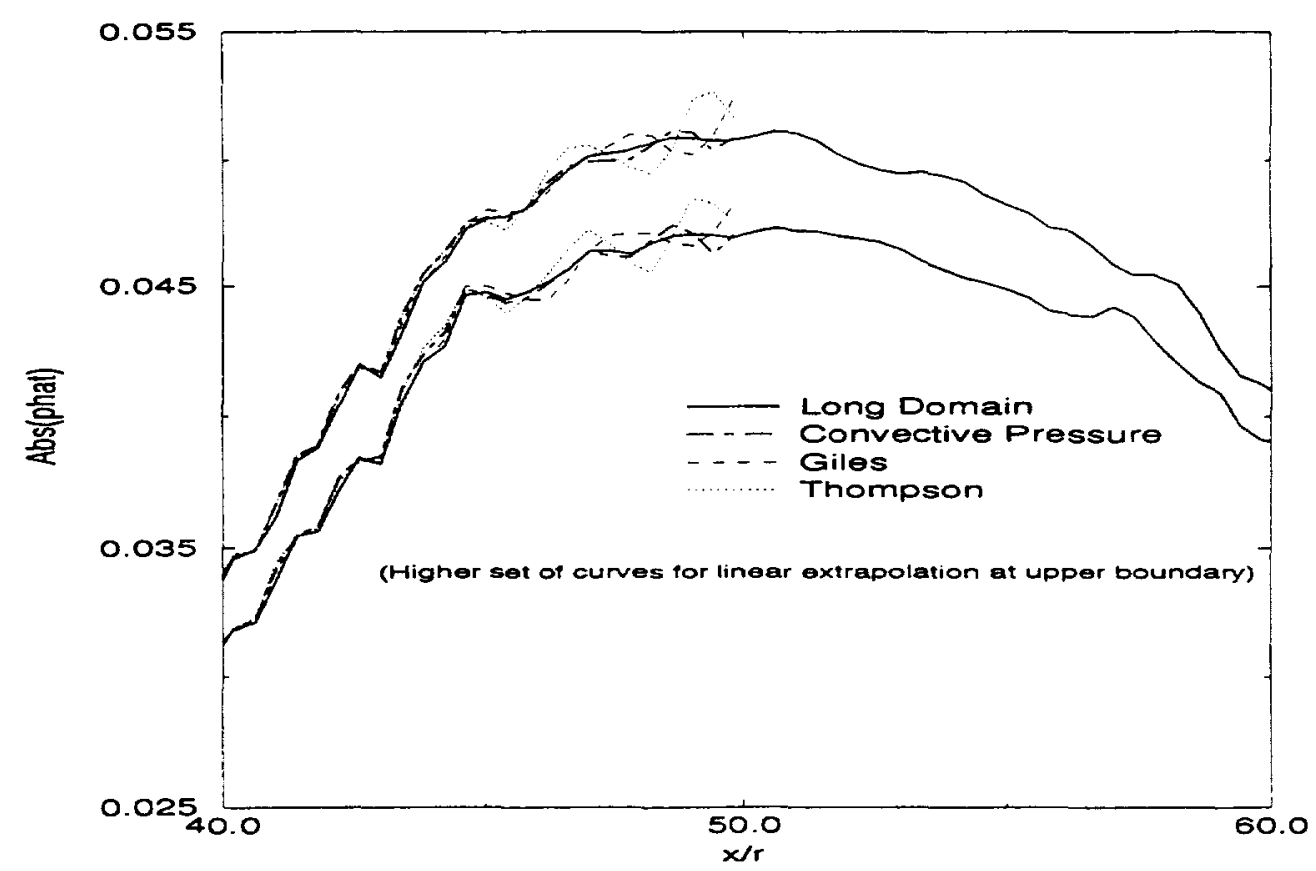

Figure 5.2: Comparison of long and short domain solutions 


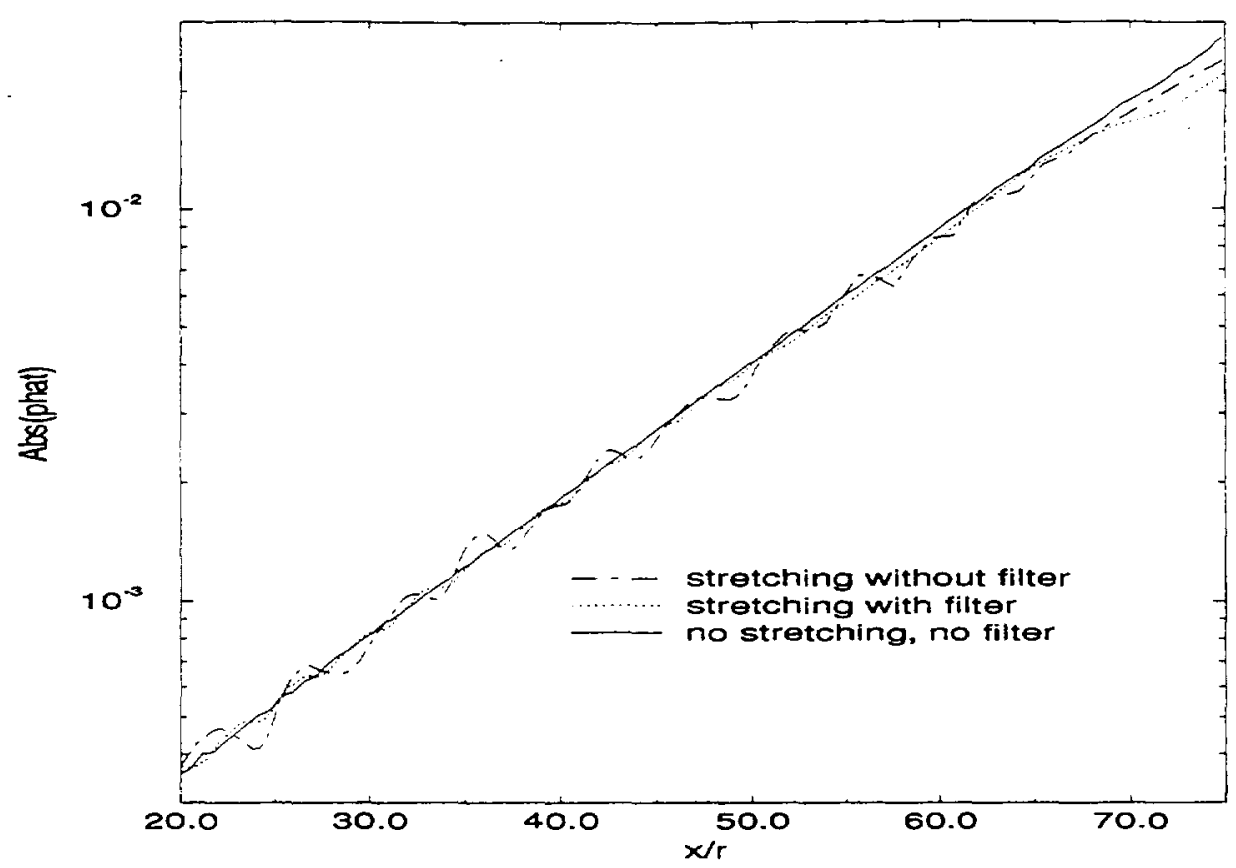

Figure 6.1: Amplitude of pressure for exit layer simulations

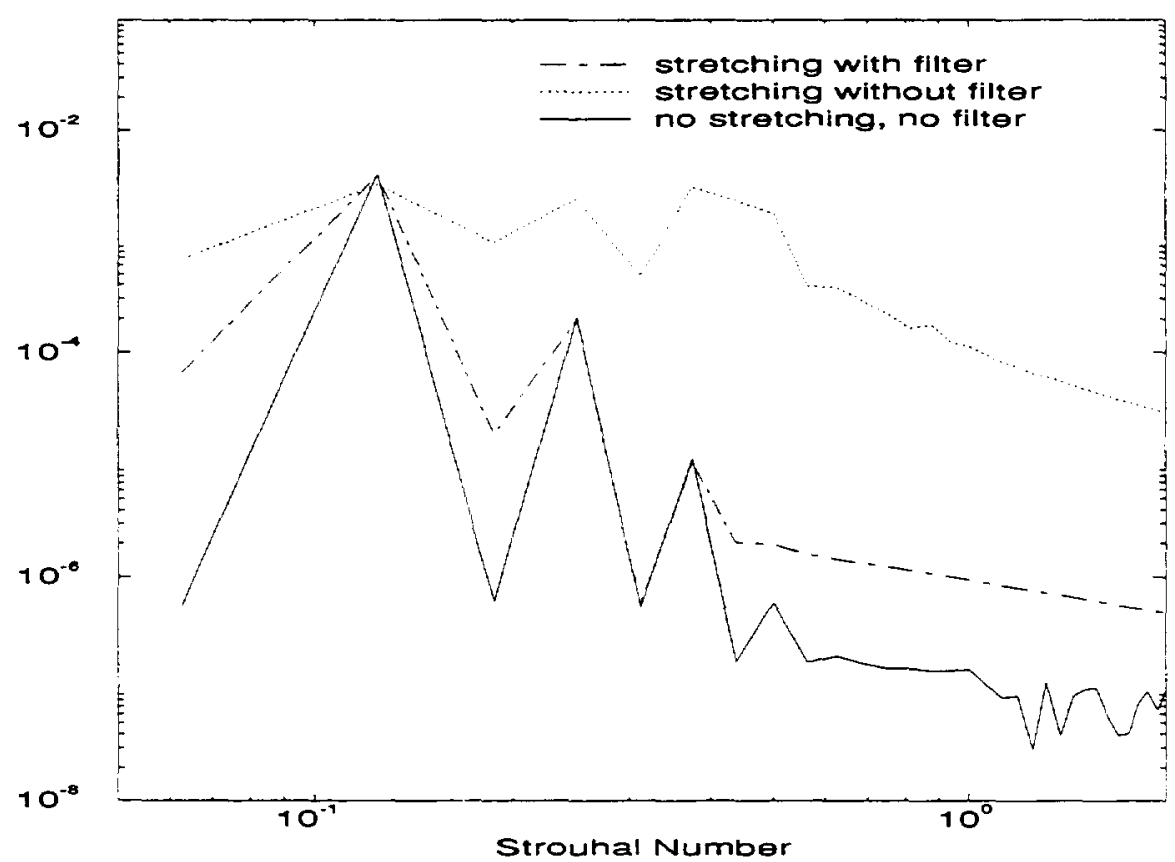

Figure 6.2: Pressure spectra al $x=50$ radii 


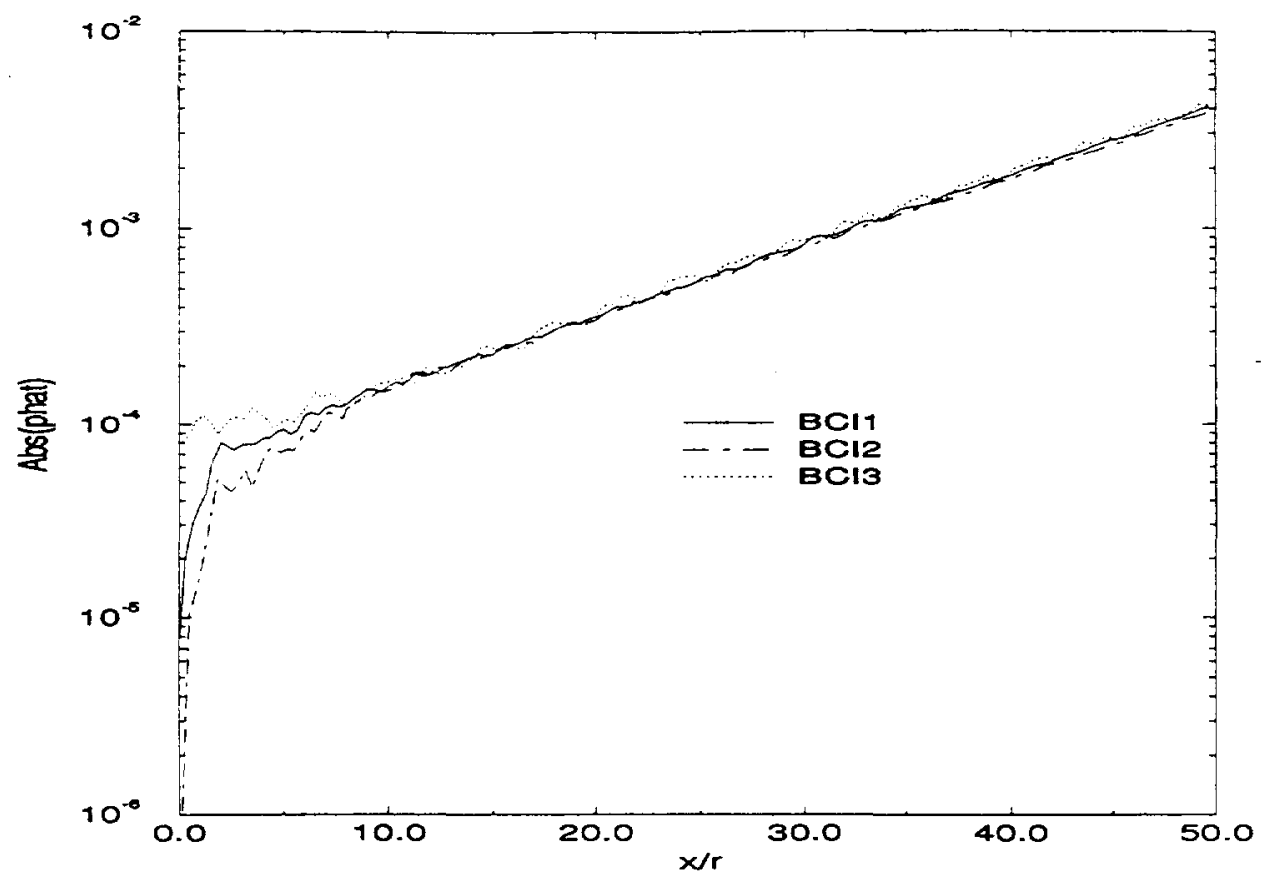

Figure 7.1: Amplitude of pressure for exit layer simulations

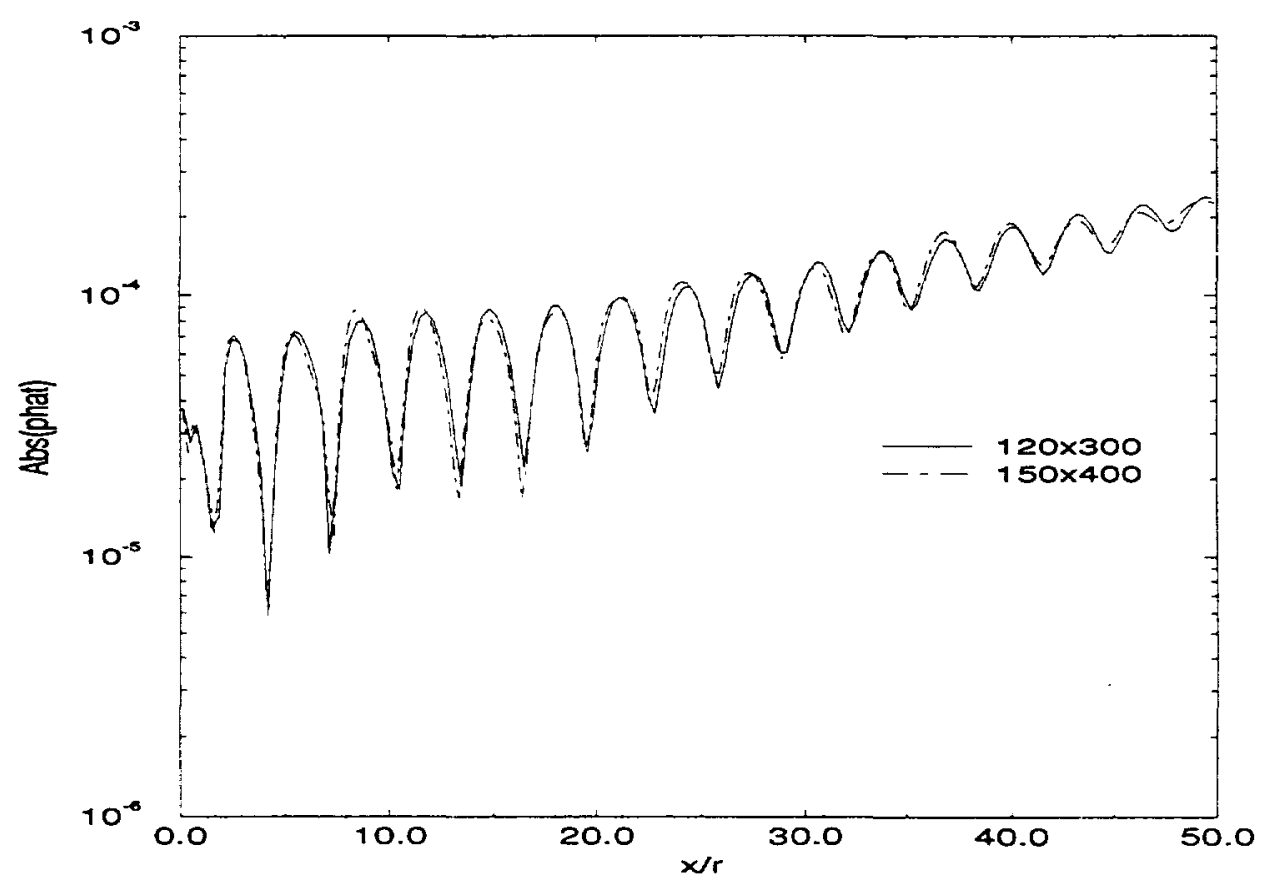

Figure 7.2: Amplitude of presssure in supersonic jet into supersonic outerflow 
Public reporting burden for this collection of information is estimated to average 1 hour per response, including the time for reviewing instructions, searching existing data sources, gathering and maintaining the data needed, and completing and reviewing the collection of information. Send comments regarding this burden estimate or any other aspect of this collection of information, including suggestions for reducing this burden, to Washington Headquarters Services, Directorate for Information Operations and Reports, 1215 Jefferson Davis Highway, Suite 1204. Artington, VA 22202-4302, and to the Office of Management and Budget, Paperwork Reduction Project (0704-018B). Washington, DC 20503.

\begin{tabular}{|l|c|r|}
\hline 1. AGENCY USE ONLY (Leave blank) & $\begin{array}{r}\text { 2. REPORT DATE } \\
\text { June } 1994\end{array}$ & $\begin{array}{r}\text { 3. REPORT TYPE AND DATES COVERED } \\
\text { Technical Memorandum }\end{array}$ \\
\hline
\end{tabular}

\section{TITLE AND SUBTITLE}

Boundary Conditions for Jet Flow Computations
5. FUNDING NUMBERS

WU-505-90-5K

M. E. Hayder and E. Turkel

\section{PERFORMING ORGANIZATION NAME(S) AND ADDRESS(ES)}

National Aeronautics and Space Administration

Lewis Research Center

Cleveland, Ohio 44135-3191
8. PERFORMING ORGANIZATION REPORT NUMBER

E-8956
9. SPONSORING/MONITORING AGENCY NAME(S) AND ADDRESS(ES)

National Aeronautics and Space Administration

Washington, D.C. 20546-0001
10. SPONSORINGMONITORING AGENCY REPORT NUMBER

NASA TM-106648

ICOMP-94-12

AIAA-94-2195

\section{SUPPLEMENTARY NOTES}

Prepared for the 25th Fluid Dynamics Conference sponsored by the American Institute of Aeronautics and Astronautics, Colorado Springs, Colorado, June 20-23, 1994. M. E. Hayder, Institute for Computational Mechanics in Propulsion, NASA Lewis Research Center (work funded by NASA Cooperative Agreernent NCC3233); E. Turkel, Institute for Computational Mechanics in Propulsion, NASA Lewis Research Center (work funded by NASA Cooperative Agreement NCC3-233) and Tel-Aviv University. Department of Mathematics, Tel-Aviv, Israel 69978. ICOMP Program Director, Louis A. Povinelli, organization code 2600, (216) 433-5818.

12a. DISTRIBUTIONAVAILABILITY STATEMENT

Unclassified -Unlimited

Subject Categories 34 and 64

\section{ABSTRACT (Maximum 200 words)}

Ongoing activities are focused on capturing the sound source in a supersonic jet through careful LES. One issue that is addressed herein is the effect of the boundary conditions, both inflow and outflow, on the predicted flow fluctuations, which represent the sound source. In this study, we examine the accuracy of several boundary conditions to determine their suitability for computations of time-dependent flows. Various boundary conditions are used to compute the flow field of a laminar axisymmetric jet excited at the inflow by a disturbance given by the corresponding eigenfunction of the linearized stability equations. We solve the full time dependent Navier-Stokes equations by a high order numerical scheme. For very small excitations, the computed growth of the modes closely corresponds to that predicted by the linear theory. We then vary the excitation level to see the effect of the boundary conditions in the nonlinear flow regime.

\section{SUBJECT TERMS}

Nonreflecting boundary conditions; Spurious oscillations

15. NUMBER OF PAGES

18

16. PRICE CODE

A03

\begin{tabular}{|c|c|}
\hline $\begin{array}{c}\text { 17. SECURITY CLASSIFICATION } \\
\text { OF REPORT } \\
\text { Unclassified }\end{array}$ & $\begin{array}{c}\text { 18. SECURTY CLASSIFICATION } \\
\text { OF THIS PAGE } \\
\text { Unclassified }\end{array}$ \\
\hline
\end{tabular}

NSN 7540-01-280-5500

19. SECURITY CLASSIFCATION OF ABSTRACT Unclassified 